\title{
Suppression of ROS generation by 4,4'-diaminodiphenylsul- fone in non-phagocytic human diploid fibroblasts
}

\author{
Sung Chun Cho, Ji Heon Rhim, Young Hoon Son, \\ Suk Jin Lee and Sang Chul Park ${ }^{1}$
}

Department of Biochemistry and Molecular Biology

Aging and Apoptosis Research Center

Seoul National University College of Medicine

Seoul 110-799, Korea

${ }^{1}$ Corresponding author: Tel, 82-2-740-8244;

Fax, 82-2-744-4534; E-mail, scpark@snu.ac.kr

DOI 10.3858/emm.2010.42.3.024

Accepted 1 February 2010

Available Online 11 February 2010

Abbreviations: CCK-8, Cell Counting Kit-8; DDS, 4,4'diaminodiphenylsulfone; DiOC6, 3,3-dihexyloxacarbocyanine; DPI, diphenylene iodonium; DPPH, 2,2-diphenyl-1-picrylhydrazyl; HDF, human diploid fibroblast; $\triangle \psi \mathrm{m}$, mitochondrial membrane potentials; NAC, acetyl-Lcysteine; NOX4, NADPH oxidase 4; ROS, reactive oxygen species

\begin{abstract}
The action mode of 4,4'-diaminodiphenylsulfone (DDS) is still under debate, although it has long been used in treatment of several dermatologic diseases including Hansen's disease. In this study, we tested the effect of DDS as an antioxidant on paraquat-induced oxidative stress in non-phagocytic human diploid fibroblasts (HDFs). Overall, preincubation of HDFs with DDS prevented the oxidative stress and the resulting cytotoxic damages caused by paraquat in these cells. The specific effects of DDS in paraquat-treated HDFs are summarized as follows: a) reducing the expression of NADPH oxidase 4 (NOX4) by inhibiting paraquat-induced activation of PKC; b) inhibiting paraquat-induced decreases in mitochondrial complex protein levels as well as in membrane potentials; c) consequently, inhibiting the generation of cytosolic and mitochondrial superoxide anions. Taken together, these findings suggest that DDS would suppress the radical generation in non-phagocytic HDFs during oxidative stress, and that DDS might have the extended potential to be used further in prevention of other oxidative stress-related pathologies.
\end{abstract}

Keywords: antioxidants; leprosy; NADPH oxidase; oxidative stress; paraquat

\section{Introduction}

4,4'-Diaminodiphenylsulfone (DDS, Dapsone), synthesized a century ago, continues to be used in the therapy of many skin diseases (Wolf et al., 2002) and has been the mainstay drug for treating patients with Hansen's disease. However, the mode of action of DDS has not been definitively established. Several studies suggested that DDS would act as a pro-oxidant and might cause hemolytic anemia (Bradshaw et al., 1997; Reilly et al., 1999). However, a recent study found little or no risk of hemolysis from topical DDS in $5 \%$ gel form (Piette et al., 2008). Furthermore, other studies suggested that DDS would act as an antioxidant rather than as a pro-oxidant (Niwa et al., 1984; Anderson et al., 1987). Thus, question whether DDS acts as a pro-oxidant or an antioxidant has not been clearly answered yet.

To answer the above question we employed paraquat in our experiment, which has been originally developed as an herbicide, and widely used as a source of oxidative stress to cells, since it generates superoxide anions via NADPH-dependent metabolic pathways. PKC is involved in the ligand-initiated assembly of NADPH oxidase (NOX) for the generation of superoxide anions (Martins et al., 2002). Recently, paraquat has been shown to induce ROS through activation of PKC-delta dependent NOX (Miller et al., 2007). In addition, mitochondria are involved in paraquat-induced oxidative stress and toxicity. Mitochondrial complex I (NADH-ubiquinone oxidoreductase) is the major mitochondrial site for superoxide production by paraquat (Cocheme et al., 2008) and paraquat significantly decreases the activity of mitochondrial complex V (Yang and Tiffani-Castiglioni, 2007). Failure of mitochondrial machinery and impairment of mitochondrial complexes by paraquat results in inhibition of electron transport with subsequent increased production of superoxide anion (Boelsterli and Lim, 2007).

Availability of the safe and effective antioxidants can possibly help prevent oxidative stress-related pathologies. The experience of long term use of DDS by Hansen's patients stimulated us to clarify the role of DDS possibly as an antioxidant and to study its underlying mechanism. For the study, the effect of DDS on paraquat-induced oxidative stress in non-phagocytic HDFs has been tested, since its 


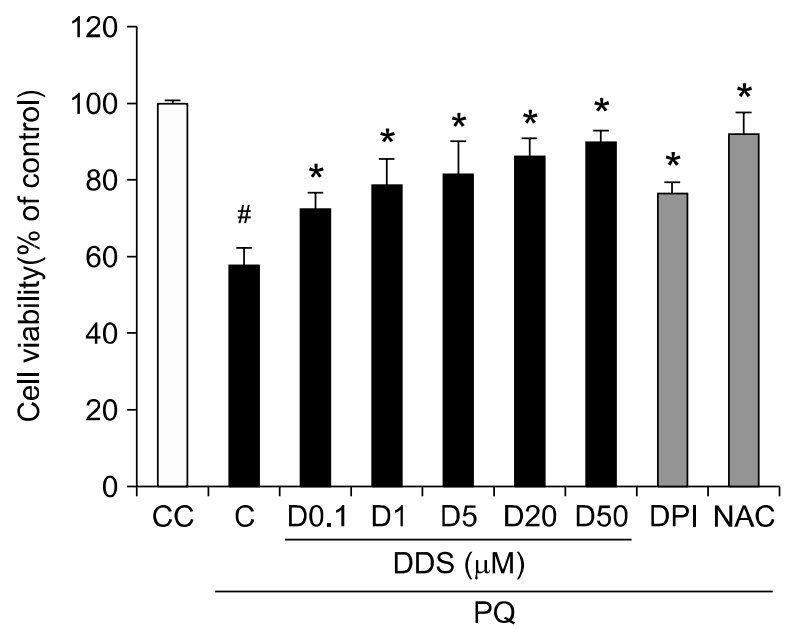

Figure 1. Effect of DDS on viability of paraquat $(P Q)$ treated HDFs. DDS was added to HDFs, and let stand for $3 \mathrm{~h}$ before treatment with $1 \mathrm{mM}$ paraquat. Cell viability was assessed after $48 \mathrm{~h}$ with a Cell Counting Kit-8. Positive controls were treated with DPI $(5 \mu \mathrm{M})$ for 30 min or with NAC (2 $\mathrm{mM}$ ) for $3 \mathrm{~h}$ prior to paraquat exposure. Data are normalized to control and the results are expressed as means \pm SE of three experiments. Results expressed as \% of control rate. Marks indicate the significant difference, $P$ $<0.005$ (\#, from untreated control cells (CC); ${ }^{*}$, from only paraquat treated control cells (C)).

role as an antioxidant during bacterial infection has been proposed in the phagocytic neutrophils (Suda et al., 2005). Through this study, we could suggest that DDS would be the effective antioxidant, which might modulate ROS generation mainly via regulation of NOX and mitochondrial dysfunction in the non-phagocytic cells.

\section{Results}

\section{DDS ameliorates paraquat-induced cytotoxicity}

To assess the cellular protective effect of DDS against paraquat-induced cytotoxicity, HDFs were pre-treated with varying concentrations of DDS $(0.1,1,5,20$, and $50 \mu \mathrm{M})$, diphenylene iodonium (DPI, $5 \mu \mathrm{M}$ ), or N-Acetyl-L-cysteine (NAC, $2 \mathrm{mM}$ ), respectively, followed by treatment of $1 \mathrm{mM}$ paraquat for $48 \mathrm{~h}$. DPI, a flavoenzyme inhibitor including NOX, and NAC, an antioxidant, were used as positive controls. Cell viability was measured with a Cell Counting Kit (CCK-8). Without pre-treatment with DDS, paraquat decreased the cell viability to about $58.8 \%$ of the control. In contrast, pre-treatment with DDS improved the cell viability against paraquat toxicity dose dependently up to $90 \%$ of the control. DPI and NAC also showed protective effects against paraquat-induced cytotoxicity and recovered the cells upto about $80-90 \%$ of the control (Figure 1).

\section{B}

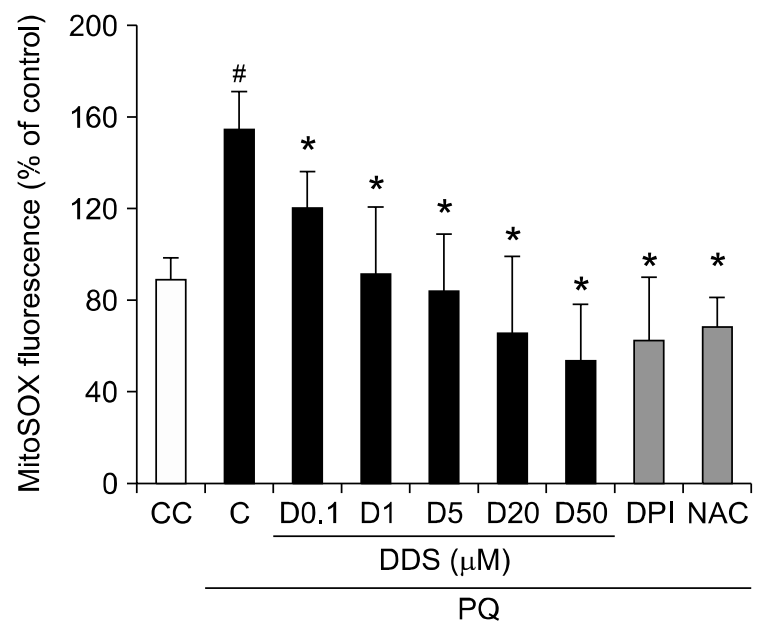

Figure 2. Effect of DDS on paraquat (PQ)-induced cytosolic or mitochondrial superoxide anion generation in HDFs. HDFs were seeded to 96 well plate for $24 \mathrm{~h}$ and pretreated with various concentration of DDS $(0.1,1,5$, and $20 \mu \mathrm{M})$, DPI $(5 \mu \mathrm{M})$ for $30 \mathrm{~min}$, and NAC $(2 \mathrm{mM})$ for $3 \mathrm{~h}$. The cells were then treated with $1 \mathrm{mM}$ paraquat for $30 \mathrm{~min}$. After paraquat treatment, the cells were incubated with the oxidant-sensitive fluorescent dyes (A) dihydroethidium (DHE) $\left(5 \mu \mathrm{M}\right.$, cytosol specific) and (B) MitoSOX Red ( $5 \mu \mathrm{M}$, mitochondria specific), respectively, for $15 \mathrm{~min}$ at $37^{\circ} \mathrm{C}$. Changes in fluorescence were monitored with a multiwell plate reader using an excitation and emission wavelength of 515 and $590 \mathrm{~nm}$ (dihydroethidium), 520 and $580 \mathrm{~nm}$ (MitoSOX Red), respectively. Results are expressed as \% of control rate. The data shown are representative of six experiments. ${ }^{\sharp} P<0.005$ relative to CC, ${ }^{*} P<0.005$ relative to $\mathrm{C}$. 
A

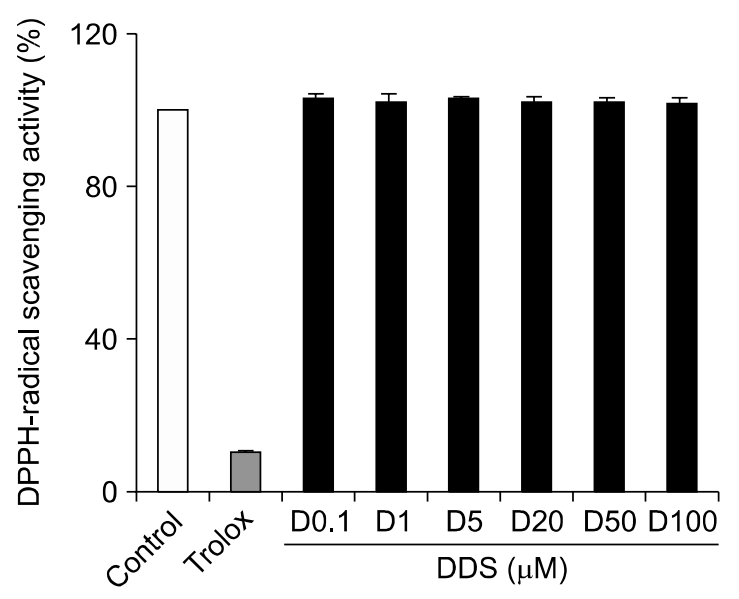

B

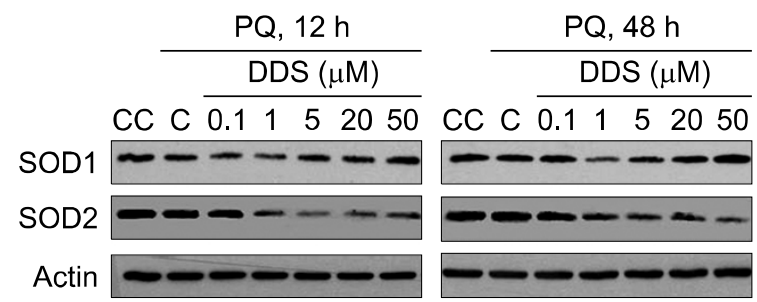

Figure 3. Effect of DDS on DPPH-radical scavenging and expression of SOD genes. (A) Radical scavenging activities of DDS in cell-free system (in vitro). The graph is obtained from studies of various concentrations of DDS and Trolox $(400 \mu \mathrm{M})$ against DPPH $(200 \mu \mathrm{M})$ radical. (B) Western blot analysis of SOD1 and SOD2 expression in DDS pretreated and paraquat $1 \mathrm{mM}$ treated HDF for $12 \mathrm{~h}$ or $48 \mathrm{~h}$.

\section{DDS inhibits paraquat-induced superoxide anion generation}

In order to monitor the paraquat-induced superoxide anion generation, we used dihydroethidium to monitor at the level of cytosol and MitoSOX Red to monitor at the level of mitochondria.

Dihydroethidium is oxidized to oxoethidium by superoxide anion, resulting in fluorescence (Miyata et al., 2005). Addition of DDS caused a dose-dependent decrease in superoxide anions as determined by dihydroethidium assay. The positive controls, DPI and NAC, also indicated the reduction in superoxide anion generation (Figure 2A). MitoSOX Red probe does not fluoresce until it enters an actively respiring cell, where it is oxidized to a fluorescent probe, selectively sequestered in the mitochondria (Esposti et al., 1999). DDS-treated HDFs showed a dose-dependent decrease in mitochondrial superoxide anions (Figure 2B). The positive controls, DPI and NAC also inhibited the generation of mitochondrial superoxide anions. These results suggest that paraquat-induced cyto- toxicity could be ameliorated by DDS via inhibition of generation of cytosolic and mitochondrial superoxide anions.

\section{DDS itself cannot scavenge radicals}

To understand how DDS protects the cells from oxidative stress, we first tested its effect on $\mathrm{DPPH}$, a stable free radical. DDS did not exert any ROS scavenging activity on DPPH in vitro, in comparison with Trolox, used as a positive antioxidant control, which showed a strong radical scavenging activity (Figure $3 \mathrm{~A}$ ). Since most antioxidants increase the oxidative defense functions with activation or induction of radical scavenging enzymes, we assumed that DDS also might induce or activate ROS scavenging enzymes. We determined the levels of superoxide dismutase 1 (SOD1) and superoxide dismutase 2 (SOD2) in HDFs in presence or absence of several concentrations of DDS. Interestingly, DDS did not increase, rather decreased the levels of SOD1 and SOD2

A

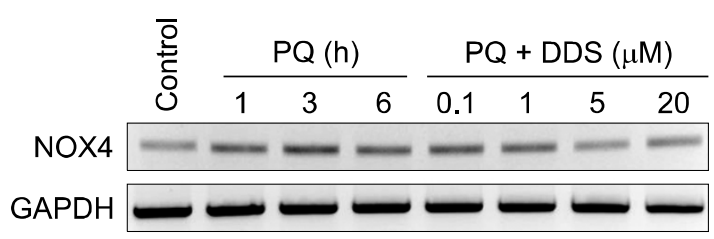

B

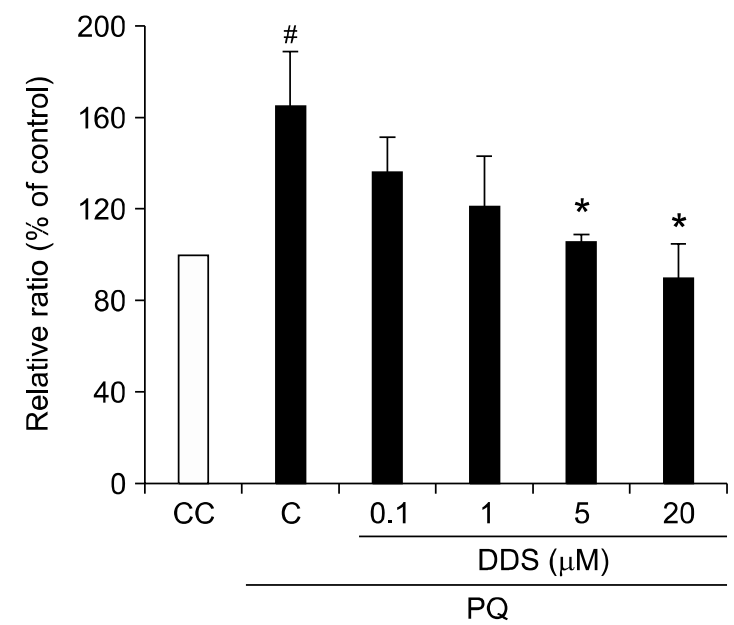

Figure 4. Effect of DDS on paraquat-induced NOX4 mRNA. (A) HDFs were pre-exposed to different concentrations of DDS for $3 \mathrm{~h}$, after which cells were exposed to $1 \mathrm{mM}$ paraquat for different time periods. NOX4 mRNA levels were determined using a RT-PCR. (B) Results are expressed as $\%$ of control rate (paraquat untreated). The data shown are representatives of three experiments from $3 \mathrm{~h}$ DDS pretreatment, followed by $3 \mathrm{~h}$ paraquat treatment. ${ }^{\#} P<0.05$ relative to $\mathrm{CC},{ }^{*} P<0.05$ relative to $\mathrm{C}$. 
A

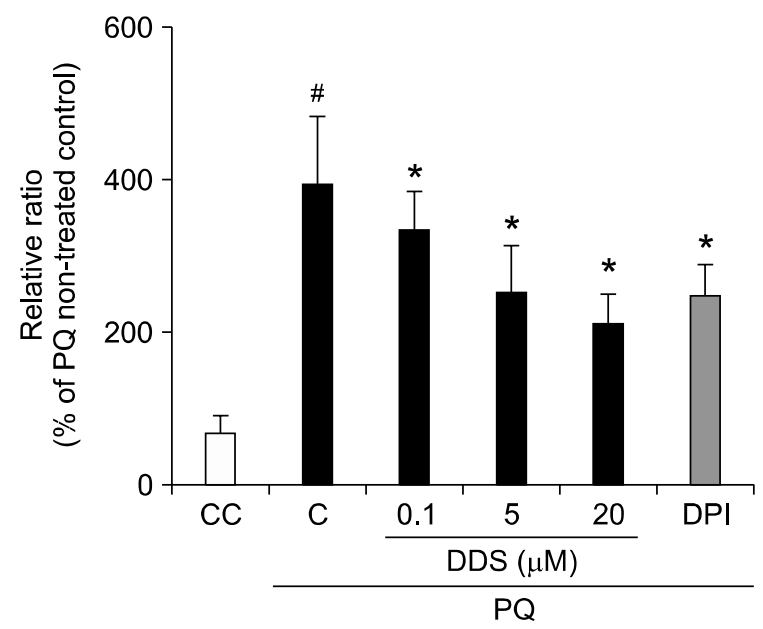

Figure 5. Effect of DDS on paraquat (PQ)-induced calcium dependent PKC activation in HDFs. (A) Effect of DDS on paraquat-induced changes in calcium levels in HDFs. HDFs were seeded to 96 well plate for $24 \mathrm{~h}$ and pretreated with various concentration of DDS and $5 \mu \mathrm{M}$ DPI for $30 \mathrm{~min}$. The cells were incubated with the intracellular calcium-indicator Fluo-4 AM at $37^{\circ} \mathrm{C}$ for $30 \mathrm{~min}$, and then exposed to 1 $\mathrm{mM}$ paraquat for several minutes. The data of 3 min paraquat treatment are representatives of three independent experiments. (B) Paraquat-induced PKC phosphorylation was determined using western blotting HDFs were first treated with DDS and then exposed to $1 \mathrm{mM}$ paraquat for $1 \mathrm{~min}$ or $5 \mathrm{~min}$. PKC phosphorylation was determined using phosphorylation-specific anti-PKC antibody. The data of PKC-pan phosphorylation (specifically serine 660) graphed is representative of three independent experiments. ${ }^{\#} P<0.05$ relative to $\mathrm{CC},{ }^{*} P<0.05$ relative to $\mathrm{C}$.

(Figure 3B), which might implicate the lesser requirement for radical scavenging activity in DDS-treated cells. These results suggest strongly that DDS would be related rather with suppression of ROS generation than with induction of ROS scavenging capacity.

\section{DDS suppresses NOX4 expression}

NOX4 is the predominant form of NOX genes in skin fibroblasts (Park et al., 2005; Rossary et al., 2007), and expression of NOX4 is associated directly with ROS generation. Down-regulation of NOX4 would result in reduction of ROS generation (Lambeth, 2004). Therefore, NOX4 has been monitored in this study. After treating cells with 1 $\mathrm{mM}$ paraquat, we subjected HDFs to RT-PCR
B
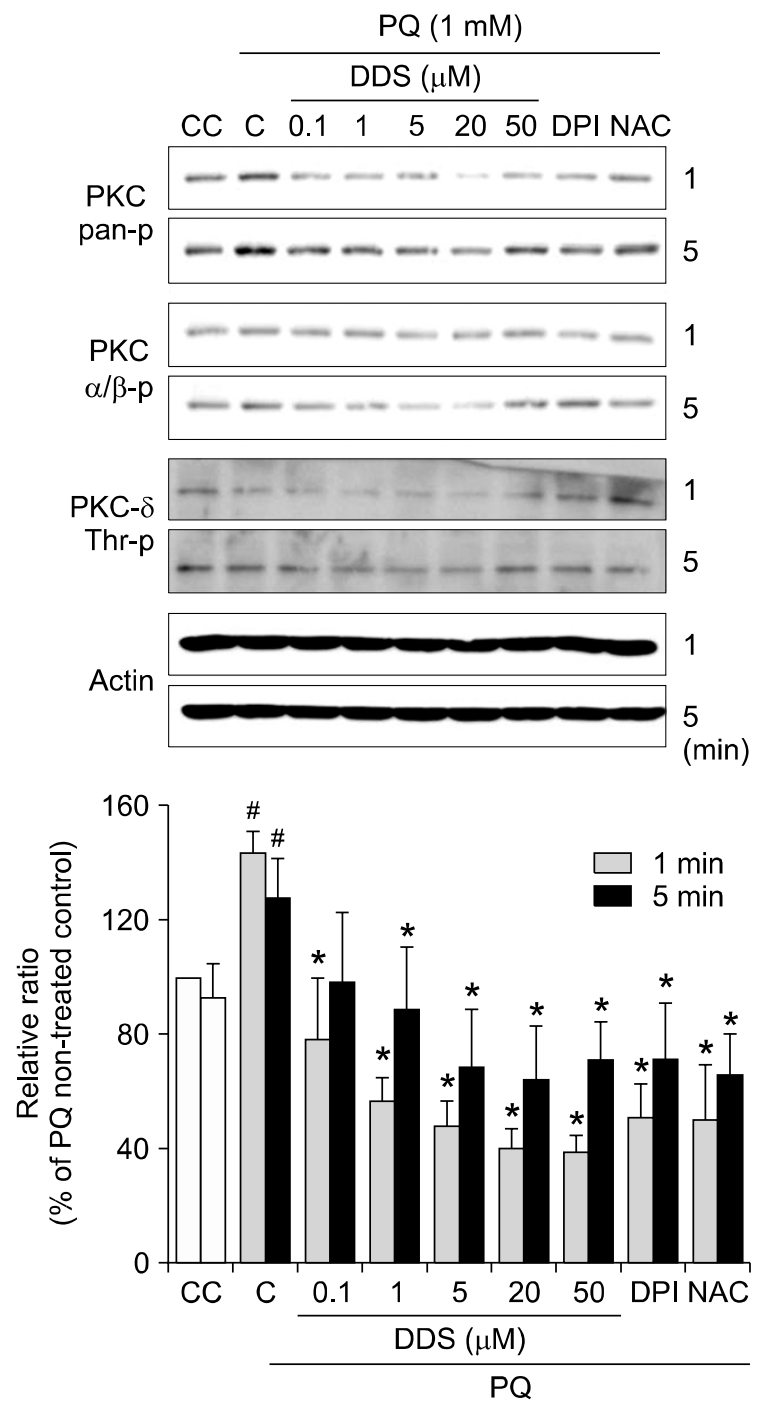

analysis of NOX4 and GAPDH. An increased level of NOX4 was observed in paraquat-treated HDFs at several indicating times $(1,3$, and $6 \mathrm{~h})$ with a peak induction at 3 hours. However, pretreatment of DDS decreased NOX4 gene expression in a dose dependent manner (Figures 4A and 4B).

\section{DDS modulates PKC activation}

It has been demonstrated that DDS can adjust intracellular calcium levels (Suda et al., 2005). In order to confirm the role of DDS in paraquat-induced calcium status, we measured calcium by Fluo-4 fluorescence in varying conditions. DDS decreased paraquat-induced calcium levels dose dependently (Figure 5A).

PKC isoforms are implicated in NOX activation 
A

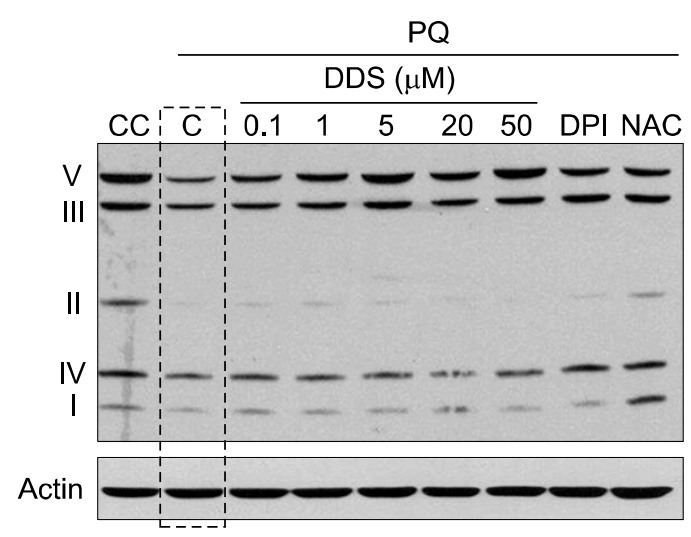

B

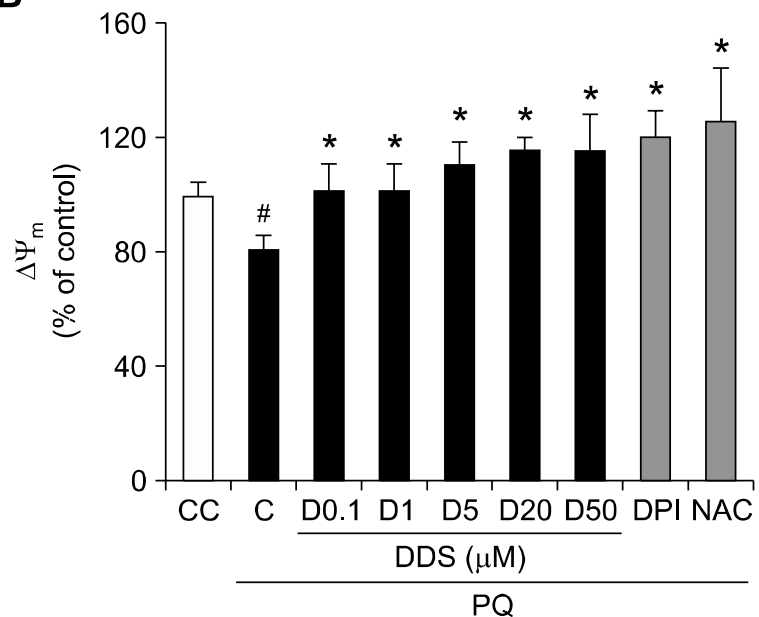

C
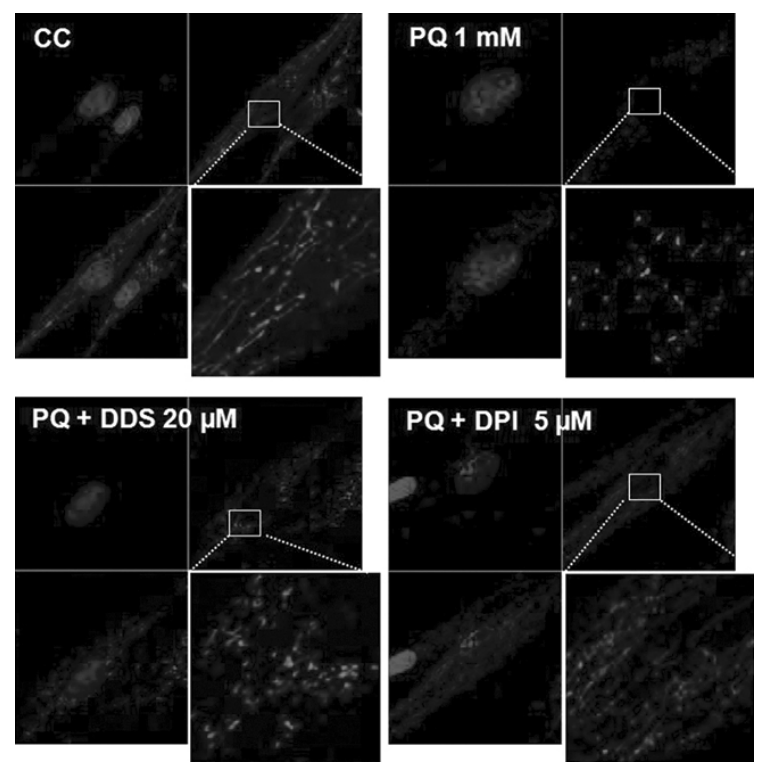

Figure 6. Effect of DDS on paraquat (PQ)-induced mitochondrial change. (A) HDFs were pretreated with DDS ( $3 \mathrm{~h})$, DPI (30 min), and NAC (3 h) and then with paraquat $1 \mathrm{mM}(24 \mathrm{~h})$. The treated HDFs were harvested for analysis of mitochondrial complex proteins, and immunoblotting with individual antibodies that recognize Complex I (20 kDa ND6 subunit), Complex II (30 kDa FeS, non-heme iron protein, SDHB), Complex III (47kDa core protein 2), Complex IV (18 kDa subunit IV, and Complex V (55 kDa subunit an ATP synthase), as described in the Methods. (B) The change of mitochondrial membrane potential $(\Delta \psi \mathrm{m})$ was examined by DiOC6. DDS, DPI, and NAC pre-treated and then paraquat $1 \mathrm{mM}(24 \mathrm{~h})$ treated HDF cells were stained $40 \mathrm{nM}$ DiOC6 for $15 \mathrm{~min}$. The result analyzed by Cary Eclipse fluorescence spectrophotometer (Varian, California) with excitation at $480 \mathrm{~nm}$ and emission at 520 $\mathrm{nm}$. (C) Mitochondria morphological change anaylsis used MitoTracker Red and confocal microscopy. HDFs growing on glass coverslips were exposed to paraquat and DDS (or DPI) as described in Methods. Cell cultures were incubated with $1 \mathrm{mM}$ paraquat for $24 \mathrm{~h}$. First, Control HDFs, second, HDFs incubated with only paraquat, third, Cell cultures co-treated with DDS $20 \mu \mathrm{M}$ and $1 \mathrm{mM}$ paraquat, and last, Cell cultures co-treated with DPI $5 \mu \mathrm{M}$ and 1 mM paraquat. (A, B, C, and D: $\times 4,000)$ Value are means \pm SEM $(n=3) .{ }^{\#} P<0.005$ relative to $C C$; ${ }^{*} P<0.005$ relative to $C$.

(Inoguchi et al., 2003). The conventional (cPKC) or calcium-dependent isoforms such as $\mathrm{PKC} \alpha, \beta \mathrm{I}, \beta \mathrm{II}$, and $\gamma$ require calcium for activation (Coppi et al., 2000). In this study with HDFs, it is confirmed that paraquat could activate only PKC $\beta$ II but not $P K C \alpha$ and PKC $\delta$. For the analysis of PKC dependent activation of NOX, we examined the effect of DDS on paraquat-induced PKC phospholylation by western blot analysis. Paraquat could activate PKC $\beta$ II (specifically via phosphorylation of ser 660 ) in HDFs, which was reduced by DDS (Figure 5B). Phospho-PKC pan antibody could detect serine 660 phosphorylation of $P K C \beta$ II, but
Phospho-PKC $\alpha / \beta$ antibody could detect PKC $\alpha / \beta$, phosphorylated at threonine 638/641.

These results implicate that DDS would suppress paraquat-induced cellular calcium signaling, followed by lowering of PKC activation with subsequent suppression of NOX4 activation, which might result in reduction of ROS generation.

\section{DDS adjusts paraquat-induced mitochondrial dysfunction}

Mitochondrial dysfunction is characterized by superoxide generation, membrane potential decrease, 
mitochondrial DNA (mtDNA) damage, and morphological changes. To understand how DDS is involved in restoring mitochondrial dysfunction, we examined the effect of DDS on paraquat-induced cell damages.

We monitored the effect of DDS on mitochondrial complex protein levels by western blot analysis. DDS restored the protein levels of all mitochondrial complexes, which had been decreased by paraquat treatment (Figure 6A). We then assessed the changes in mitochondrial membrane potentials by DiOC6 fluorescence. Mitochondrial membrane potential in HDFs decreased to $76.8 \%$ of the control after treatment with $1 \mathrm{mM}$ paraquat for 24 h. Pre-treatment with several concentrations of DDS for $3 \mathrm{~h}$ or $5 \mu \mathrm{M}$ DPI for 30 min significantly protected HDFs from paraquat-induced decrease in mitochondrial membrane potential (Figure 6B).

In normal HDFs, mitochondria exhibit elongated patterns (Figure 6C, first). Cells are defined MitoTracker Red positive (MT+), if well defined fluorescent points corresponding to mitochondria are observed, and MitoTracker Red negative (MT-) when a diffuse cytoplasmic staining is observed (Matylevitch et al., 1998). HDFs, incubated with 1 $\mathrm{mM}$ paraquat for $24 \mathrm{~h}$, showed spotting pattern on staining, and the disappearance of the MT (+) cell morphology (Figure 6C, second). When HDFs were co-treated with DDS (or DPI) and paraquat, there was a partial recovery of original mitochondrial morphology (Figure 6C, third and fourth). Because MitoTracker Red dye could be used for monitoring the mitochondrial membrane potential (McCarthy et al., 2004), these results also support the effect of DDS on paraquat-induced mitochondrial membrane potential collapses.

\section{Discussion}

Long-term administration of DDS at the standard dose (100 mg/day) in patients with Hansen's disease usually results in little or no clinically significant side effects. Administration of DDS, 100 $\mathrm{mg} /$ day, for 6-28 days to patients results in 1-20 $\mu \mathrm{M}$ of plasma DDS concentration (Zone, 1991). A recent study evaluating the risk of hemolysis reported that the topical use of DDS at $5 \% \mathrm{gel}$ would be safe, and DDS levels of plasma could reach about $5 \mathrm{ng} / \mathrm{ml}(20 \mu \mathrm{M})$ (Piette et al., 2008).

Recently it has been reported that DDS suppresses the production of $\mathrm{O}_{2}{ }^{\cdot-}$ in phagocytic neutrophils (Suda et al., 2005). Thereby, we asked the question whether its effect as an antioxidant could be reproduced in non-phagocytic cells. ROS generation in non-phagocytic cells is much lower than in phagocytic cells, and its implication might be distinct from bacterial killing. For the study on the cellular effect of DDS in non-phagocytic HDFs, we adopted the paraquat-induced oxidative stress cytotoxic model, since the mechanism of paraquatinduced cytotoxicity has been well associated with oxygen radical generation.

In this study, we tried to test the possibility that DDS might act as an effective blocker of radical generation, not only in phagocytic cells but also in non-phagocytic cells. Initially, the genuine radical scavenging activity of DDS has been tested on DPPH, a stable free radical (Dinis et al., 1994; Wang et al., 1998). Antioxidants react with DPPH and convert it to 1,1-diphenyl-2-(2,4,6-trinitrophenyl) hydrazine, indicating their scavenging potentials. But in this study, DDS itself could not scavenge DPPH radicals in vitro, in contrast to the strong scavenging activity of Trolox, a positive control for radical scavenging (Figure $3 A$ ). Then the effect of DDS on expression levels of radical scavenging enzymes, SOD1 and SOD2 has been assessed. Cytosolic copper-zinc SOD (SOD1) and mitochondrial manganese-SOD (SOD2) reduce superoxide anion to hydrogen peroxide $\left(\mathrm{H}_{2} \mathrm{O}_{2}\right)$ and molecular oxygen (Zelko et al., 2002). Interestingly, DDS could reduce the expression of both enzymes in HDFs rather than their induction (Figure 3B). These data strongly suggest that the antioxidant function of DDS would not be directly related to the radical scavenging system, neither by direct radical scavenging activity nor by induction of radical scavenging enzymes.

Therefore, we focused on the role of DDS in modulation of radical generation rather than in radical scavenging. Paraquat-induced oxidative stress, mainly mediated by activation of the NOX, but not by activation of xanthine oxidase (Miller et al., 2007), is accompanied by mitochondrial dysfunction (Thakar et al., 1988; Tomita, 1991). NOXs utilize molecular oxygen and NADPH as substrates and FAD as a coenzyme. NOX enzymes are involved in regulation of a wide range of physiological functions including cellular survival, differentiation, proliferation, $\mathrm{Ca}^{2+}$ signaling and migration (Lambeth, 2004). NOX4, the major NOX form present in skin fibroblasts, generates substantial amounts of ROS (Park et al., 2005; Rossary et al., 2007) and is increased by TGF $\beta$ stimulation (Cucoranu et al., 2005; Sturrock et al., 2006) and by angiotensin stimulation of mesangial cells in the kidney (Gorin et al., 2003). In this study, NOX4 mRNA level was found to be increased by paraquat treatment, which was suppressed by DDS (Figure 4).

DDS was suggested to be involved in calcium modulation (Suda et al., 2005). However, no data 
are available about its effect on paraquat-modulated calcium levels in HDFs. In this study, it was confirmed that paraquat would induce the intracellular calcium and that this increase could be reversed by DDS (Figure 5B). The increased calcium might activate the calcium-dependent events such as PKC activation, required for NOX activation. The association of paraquat-induced superoxide anion generation with activation of PKC is well established (Martins et al., 2002; Miller et al., 2007). Therefore, it was asked whether DDS could modulate PKC activation. Our results indicated that DDS could inhibit paraquat-induced PKC $\beta$ II phosphorylation at serine 660 in HDFs (Figure 5B). Taken together, these findings suggest that DDS would restore the intracellular $\mathrm{Ca}^{2+}$ levels, followed by inhibition of PKC-mediated activation of NOX, resulting in suppression of superoxide anion generation in paraquat-treated HDFs.

Paraquat-induced mitochondrial dysfunction is characterized by high superoxide production, reduced membrane potential, mitochondrial DNA damage and morphological changes. Paraquat is used in experimental models of Parkinson disease to generate mitochondrial oxidative damage (Thiruchelvam et al., 2000). Thereby, antioxidants are investigated for possible regulators of mitochondrial superoxide anion generation and consequently for treatment of neurodegenerative diseases. Our study demonstrated that DDS might attenuate the paraquat-induced mitochondrial abnormalities in HDFs. Not unexpectedly, DPI, a large-spectrum inhibitor of electron transporters including various NOX enzymes and mitochondrial oxidase (Li et al., 1998), inhibited paraquat-induced mitochondrial toxicity. DDS thus emerges as a possible novel efficient inhibitor of paraquat toxicity.

In summary, we have shown here that DDS inhibits paraquat-induced oxidative stress and mitochondrial toxicity in HDFs through inhibition of NOX4 activation by modulation of $\mathrm{Ca}^{2+}$ level and the consequent regulation of PKC activity. Further studies are required to confirm the effectiveness of DDS in prevention of other oxidative stress-related pathologic conditions in general. In the mean time, it might be tentatively proposed that DDS would be the novel potential agent for mitigating or minimizing oxidative stress via its ability to suppress the generation of oxygen radicals.

\section{Methods}

\section{Chemicals}

The following are the sources of various chemicals and reagents used: 4,4'-diaminodiphenylsulfone (DDS, dapsone) was from Taekeuk pharmaceuticals (Seoul, Korea); Paraquat, 2,2-diphenyl-1-picrylhydrazyl (DPPH), Trolox, diphenylene iodonium (DPI), and N-Acetyl-L-cysteine (NAC) were from Sigma (St. Louis, MO); Cell Counting Kit (CCK-8) was from Dojindo Laboratory (Japan), and MS601 (a Total OXPHOS Complexe Detection Kit, antibody) was from Mitoscience (Eugene, OR). SOD1 and SOD2 antibodies were from AbFrontier (Seoul, Korea); Trizol reagent was from MRC (Cincinnati), and dihydroethidium, MitoSOX Red, Fluo-4 AM, MitoTracker Red CMXRos, and 3, 3-dihexyloxacarbocyanine (DiOC6) were from Molecular Probes (Eugene, OR).

\section{Cell culture and assessment of cell viability}

Primary HDFs were isolated from newborn foreskins, as described (Boyce and Ham, 1983). Cells were maintained in DMEM containing $10 \%$ FBS and antibiotics. Cell viability assessment was performed using Cell Counting Kit (CCK-8) as required in Dojindo Laboratory technical manual. HDFs were seeded and grown for $24 \mathrm{~h}$ in 96-well plates prior to any treatment. HDFs were pre-treated with DDS for $3 \mathrm{~h}$, and then treated with paraquat $(1 \mathrm{mM})$ for 48 $\mathrm{h}$ and $10 \mu \mathrm{l} \mathrm{CCK}-8$ were directly added to each well, and incubated for $3 \mathrm{~h}$ at $37^{\circ} \mathrm{C}$ under $5 \% \mathrm{CO}_{2}$. The results were expressed relative to the control values specified in each experiment and were subjected to statistical analysis.

\section{Determination of radical scavenging activity}

The radical scavenging activity was determined by reduction of 2,2-diphenyl-1-picrylhydrazyl (DPPH) in methanol. Free radical scavenging effect of DDS was determined as previously described (Mellors and Tappel, 1966), using Trolox as a reference antioxidant. In brief, 0.5 $\mathrm{ml}$ of methanol containing different concentrations (0.1-100 $\mu \mathrm{M})$ of DDS and Trolox $(400 \mu \mathrm{M})$ were mixed in the test tube with $2.5 \mathrm{ml}$ methanol containing $200 \mu \mathrm{M} \mathrm{DPPH}$, respectively. DPPH is a stable free radical with a deep purple color in alcohol solution and has a peak absorbance at $517 \mathrm{~nm}$, but becomes pale yellow when trapped by an antioxidant. The reaction mixture was kept in dark at room temperature for $30 \mathrm{~min}$ and the absorbance was monitored at $517 \mathrm{~nm}$. The free radical scavenging activity was approximated using the following equation: $\%$ inhibition = [(absorbance of control-absorbance of test sample)/absorbance of control] $\times 100 \%$.

\section{RT-PCR detection of NOX and GAPDH}

Total RNA of HDFs was prepared with Trizol reagent (MRC Cincinnati), and RT-PCR for NOX4 and GAPDH were performed with Biometra $T$ Gradient PCR (Biometra, Goettingen, Germany) using specific primers: 5'-GGTCCTTTTGGAAGTCCATTTGAGG-3' (forward primer) and 5'-CACAGCTGATTGATTCCGCTGAG-3' (reverse primer) for NOX4; 5'-ACCACAGTCCATGCCATCAC-3' (forward primer) and 5'-TCCACCACCCTGTTGCTGTA-3' (reverse primer) for GAPDH. PCR-amplified DNA was separated by $1.2 \%$ agarose gel electrophoresis. 


\section{Monitoring of the intracellular superoxide anion}

HDFs were seeded to 96 well plate and were subjected to pretreatment with various concentration of DDS, DPI (5 $\mu \mathrm{M}, 30 \mathrm{~min})$, and NAC (2 $\mathrm{mM})$ for $3 \mathrm{~h}$ respectively. HDFs were then treated with $1 \mathrm{mM}$ paraquat for $30 \mathrm{~min}$, and then incubated with $5 \mu \mathrm{M}$ dihydroethidium for $15 \mathrm{~min}$ at $37^{\circ} \mathrm{C}$ in dark. The cells were rinsed twice with DPBS, and then ethidium-DNA fluorescence was immediately measured with a Cary Eclipse fluorescence spectrophotometer (Varian, California) with an excitation wavelength at $515 \mathrm{~nm}$ and an emission wavelength at $590 \mathrm{~nm}$.

\section{Detection of mitochondrial ROS}

Mitochondrial ROS production was detected using MitoSOX Red. HDFs were treated with $1 \mathrm{mM}$ paraquat for 30 min under each condition (DDS, DPI, and NAC pre-treated), and then MitoSOX Red was added at a final concentration of $5 \mu \mathrm{M}$. After incubation at $37^{\circ} \mathrm{C}$ for further $15 \mathrm{~min}$, the cells were washed twice with DPBS. The fluorescence was immediately measured with a Cary Eclipse fluorescence spectrophotometer (Varian, California) with an excitation wavelength at $515 \mathrm{~nm}$ and an emission wavelength at $590 \mathrm{~nm}$.

\section{Western blot analysis for PKC and mitochondrial complexes}

Lysates of DDS and $1 \mathrm{mM}$ paraquat treated HDFs were boiled in sample buffer (50 mM Tris- $\mathrm{HCl}$ (pH 6.8), $2 \%$ SDS, $0.14 \mathrm{M}$ 2-mercaptoethanol, $10 \%$ glycerol, and $0.001 \%$ bromphenol blue) and were subjected to electrophoresis. After transfer to nitrocellulose, western blots were carried out with the respective antibodies to MS601 (Total OXPHOS Complexes Detection Kit, MitoSciences, containing antibodies against SDH30 subunit of complex II $(5 \mu \mathrm{g} / \mathrm{ml})$, Core2 subunit of complex III $(0.2 \mu \mathrm{g} / \mathrm{ml})$, COXII subunit of complex IV $(2 \mu \mathrm{g} / \mathrm{ml})$, and subunit $\alpha$ of F1-ATPase of complex V $(0.2 \mu \mathrm{g} / \mathrm{ml})$ ), PCKpan-p ( $\beta \mathrm{Il}$ ser660), PCK $\alpha / \beta-p$ (Thr638/641), PKC $\delta-p$ (Phospho-PKC antibody Sampler Kit, Cell Signaling Technology), and $\beta$-Actin (Sigma). Detection was carried out using HRP-conjugated secondary antibodies (Zymed) with ECL (Pierce), visualized by exposing blots to autoradiographic film (Image Reader, LAS-3000, Fujifilm, Japan).

\section{Measurement of intracellular $\mathrm{Ca}^{2+}$}

Intracellular free $\mathrm{Ca}^{2+}$ levels were assessed semi-quantitatively by loading cells with $5 \mu \mathrm{M}$ of the cell-permeate fluorescent calcium indicator Fluo-4 AM at $37^{\circ} \mathrm{C}$ for $30 \mathrm{~min}$ and at the end of incubation, cells were further incubated for additional $30 \mathrm{~min}$ at room temperature to allow complete deesterification of intracellular Fluo-4 AM. After rinsing cells once with $20 \mathrm{mM}$ HEPES (pH7.4), $100 \mu \mathrm{l}$ HBSS was added into each well. The fluorescent signal was measured at the indicated times using a multi-well fluorescence plate reader (Spectrafluor Plus, Tecan, Switzerland) with an excitation wavelength at $494 \mathrm{~nm}$ and an emission wavelength at $516 \mathrm{~nm}$ (Minta et al., 1989). Data were presented by relative fluorescence units
(RFUs).

\section{Assay for mitochondrial membrane potential}

Changes in mitochondrial membrane potential $(\Delta \psi \mathrm{m})$ were determined by staining the cells with mitochondrial specific fluorescent cationic dye, DiOC6, with fluorescence spectrophotometer (Quillet-Mary et al., 1997; Hishita et al., 2001). $40 \mathrm{nM}$ DiOC6 was added to the incubation medium for 15 min before the end of the treatment at $37^{\circ} \mathrm{C}$. Then after washing, the cells were analyzed by Cary Eclipse fluorescence spectrophotometer (Varian, California) with excitation at $480 \mathrm{~nm}$ and emission at $520 \mathrm{~nm}$.

\section{MitoTracker Red staining}

HDFs, plated in 24-well plates and treated as above for 24 $\mathrm{h}$, were incubated with the mitochondrial dye MitoTracker Red CMXRos $(50 \mathrm{nM})$ for $30 \mathrm{~min}$ at $37^{\circ} \mathrm{C}$. These dye labels mitochondria with intact transmembrane potential and thus the level of staining would serve as an index of mitochondrial function. After this incubation and rinsing with PBS, the cells were fixed with $4 \%$ paraformaldehyde overnight at $4^{\circ} \mathrm{C}$. After washing with PBS, the cells were incubated for $1 \mathrm{~h}$ at room temperature with blocking solution ( $2 \%$ bovine serum albumin in PBS). The HDFs were then incubated for $10 \mathrm{~min}$ at room temperature with DAPI. After further washes, coverslips were applied, and the cells were visualized under a fluorescent microscope.

\section{Statistical analysis}

The statistical comparisons for continuous variables were performed using the t-test or analysis of variance (ANOVA) test where appropriate. SPSS software version 15.0 (SPSS, Inc., Chicago, IL) was used for all statistical analyses.

\section{Acknowledgements}

This study has been supported by the grants from the Aging and Apoptosis Research Center of the Korean Science and Engineering Foundation (R11-2002-09705-001-0), Research Program of Cancer and Aging from KOSEF and KRIBB Research Institute Program, and the SNU BK21 Program from Ministry of Education, Science and Technology to Cho SC, Son YH and Lee SJ.

\section{Rerefences}

Anderson R, Theron AJ, Ras GJ. Regulation by the antioxidants ascorbate, cysteine, and dapsone of the increased extracellular and intracellular generation of reactive oxidants by activated phagocytes from cigarette smokers. Am Rev Respir Dis 1987;135:1027-32

Boelsterli UA, Lim PL. Mitochondrial abnormalities--a link to idiosyncratic drug hepatotoxicity? Toxicol Appl Pharmacol 2007;220:92-107

Boyce ST, Ham RG. Calcium-regulated differentiation of 
normal human epidermal keratinocytes in chemically defined clonal culture and serum-free serial culture. J Invest Dermatol 1983;81:33s-40s

Bradshaw TP, McMillan DC, Crouch RK, Jollow DJ. Formation of free radicals and protein mixed disulfides in rat red cells exposed to dapsone hydroxylamine. Free Radic Biol Med 1997;22:1183-93

Cocheme HM, Murphy MP. Complex I is the major site of mitochondrial superoxide production by paraquat. J Biol Chem 2008;283:1786-98

Coppi AA, Lesniak J, Zieba D, Schanne FA. The effects of lead on PKC isoforms. Ann N Y Acad Sci 2000;919:304-6

Cucoranu I, Clempus R, Dikalova A, Phelan PJ, Ariyan S, Dikalov $S$, Sorescu D. NAD $(P) H$ oxidase 4 mediates transforming growth factor-beta1-induced differentiation of cardiac fibroblasts into myofibroblasts. Circ Res 2005;97: 900-7

Dinis TC, Maderia VM, Almeida LM. Action of phenolic derivatives (acetaminophen, salicylate, and 5-aminosalicylate) as inhibitors of membrane lipid peroxidation and as peroxyl radical scavengers. Arch Biochem Biophys 1994; 315:161-9

Esposti MD, Hatzinisiriou I, McLennan H, Ralph S. Bcl-2 and mitochondrial oxygen radicals. New approaches with reactive oxygen species-sensitive probes. J Biol Chem 1999; 274:29831-7

Gorin Y, Ricono JM, Kim NH, Bhandari B, Choudhury GG, Abboud HE. Nox4 mediates angiotensin II-induced activation of Akt/protein kinase B in mesangial cells. Am J Physiol Renal Physiol 2003;285:F219-29

Hishita T, Tada-Oikawa S, Tohyama K, Miura Y, Nishihara T, Tohyama Y, Yoshida Y, Uchiyama T, Kawanishi S. Caspase-3 activation by lysosomal enzymes in cytochrome c-independent apoptosis in myelodysplastic syndrome-derived cell line P39. Cancer Res 2001;61:2878-84

Inoguchi $\mathrm{T}$, Sonta $\mathrm{T}$, Tsubouchi $\mathrm{H}$, Etoh $\mathrm{T}$, Kakimoto $\mathrm{M}$, Sonoda N, Sato N, Sekiguchi N, Kobayashi K, Sumimoto H, Utsumi H, Nawata H. Protein kinase C-dependent increase in reactive oxygen species (ROS) production in vascular tissues of diabetes: role of vascular $\mathrm{NAD}(\mathrm{P}) \mathrm{H}$ oxidase. J Am Soc Nephrol 2003;14:S227-32

Lambeth JD. NOX enzymes and the biology of reactive oxygen. Nat Rev Immunol 2004;4:181-9

Li JM, Shah AM. Intracellular localization and preassembly of the NADPH oxidase complex in cultured endothelial cells. J Biol Chem 2002;277:19952-60

Li Y, Trush MA. Diphenyleneiodonium, an NAD(P)H oxidase inhibitor, also potently inhibits mitochondrial reactive oxygen species production. Biochem Biophys Res Commun 1998; 253:295-9

Martins Chaves M, Prates Rodrigues AL, Pereira dos Reis A, Gerzstein NC, Nogueira-Machado JA. Correlation between NADPH oxidase and protein kinase $C$ in the ROS production by human granulocytes related to age. Gerontology 2002;48:354-9

Matylevitch NP, Schuschereba ST, Mata JR, Gilligan GR,
Lawlor DF, Goodwin CW, Bowman PD. Apoptosis and accidental cell death in cultured human keratinocytes after thermal injury. Am J Pathol 1998;153:567-77

McCarthy S, Somayajulu M, Sikorska M, Borowy-Borowski $\mathrm{H}$, Pandey S. Paraquat induces oxidative stress and neuronal cell death; neuroprotection by water-soluble Coenzyme Q10. Toxicol Appl Pharmacol 2004;201:21-31

Mellors A, Tappel AL. The inhibition of mitochondrial peroxidation by ubiquinone and ubiquinol. J Biol Chem 1966; 241:4353-6

Miller RL, Sun GY, Sun AY. Cytotoxicity of paraquat in microglial cells: Involvement of PKCdelta- and ERK1/2-dependent NADPH oxidase. Brain Res 2007;1167: 129-39

Minta A, Kao JP, Tsien RY. Fluorescent indicators for cytosolic calcium based on rhodamine and fluorescein chromophores. J Biol Chem 1989;264:8171-8

Miyata K, Rahman M, Shokoji T, Nagai Y, Zhang GX, Sun GP, Kimura S, Yukimura T, Kiyomoto H, Kohno M, Abe Y, Nishiyama A. Aldosterone stimulates reactive oxygen species production through activation of NADPH oxidase in rat mesangial cells. J Am Soc Nephrol 2005;16:2906-12

Niwa Y, Sakane T, Miyachi Y. Dissociation of the inhibitory effect of dapsone on the generation of oxygen intermediates--in comparison with that of colchicine and various scavengers. Biochem Pharmacol 1984;33:2355-60

Park HS, Jin DK, Shin SM, Jang MK, Longo N, Park JW, Bae DS, Bae YS. Impaired generation of reactive oxygen species in leprechaunism through downregulation of Nox4. Diabetes 2005;54:3175-81

Piette WW, Taylor S, Pariser D, Jarratt M, Sheth P, Wilson D. Hematologic safety of dapsone gel, $5 \%$, for topical treatment of acne vulgaris. Arch Dermatol 2008;144:1564-70

Quillet-Mary A, Jaffrezou JP, Mansat V, Bordier C, Naval J, Laurent $\mathrm{G}$. Implication of mitochondrial hydrogen peroxide generation in ceramide-induced apoptosis. J Biol Chem 1997;272:21388-95

Reilly TP, Woster PM, Svensson CK. Methemoglobin formation by hydroxylamine metabolites of sulfamethoxazole and dapsone: implications for differences in adverse drug reactions. J Pharmacol Exp Ther 1999;288:951-9

Rossary A, Arab K, Steghens JP. Polyunsaturated fatty acids modulate NOX 4 anion superoxide production in human fibroblasts. Biochem J 2007;406:77-83

Sturrock A, Cahill B, Norman K, Huecksteadt TP, Hill K, Sanders K, Karwande SV, Stringham JC, Bull DA, Gleich M, Kennedy TP, Hoidal JR. Transforming growth factor-beta1 induces Nox4 $\mathrm{NAD}(\mathrm{P}) \mathrm{H}$ oxidase and reactive oxygen species-dependent proliferation in human pulmonary artery smooth muscle cells. Am J Physiol Lung Cell Mol Physiol 2006;290:L661-73

Suda T, Suzuki Y, Matsui T, Inoue T, Niide O, Yoshimaru T, Suzuki H, Ra C, Ochiai T. Dapsone suppresses human neutrophil superoxide production and elastase release in a calcium-dependent manner. $\mathrm{Br} \mathrm{J}$ Dermatol 2005;152: 887-95 
Thakar JH, Hassan MN. Effects of 1-methyl-4-phenyl1,2,3,6-tetrahydropyridine (MPTP), cyperquat (MPP+) and paraquat on isolated mitochondria from rat striatum, cortex and liver. Life Sci 1988;43:143-9

Thiruchelvam M, Richfield EK, Baggs RB, Tank AW, Cory-Slechta DA. J Neurosci 2000;20:9207-14

Tomita M. Comparison of one-electron reduction activity against the bipyridylium herbicides, paraquat and diquat, in microsomal and mitochondrial fractions of liver, lung and kidney (in vitro). Biochem Pharmacol 1991;42:303-9

Wang MF, Li JG, Rangarajan M, Shao Y, LaVoie EJ, Huang TC, Ho CT. Antioxidantive phenolic compounds from sage (Salvia officinalis). J Agric Food Chem 1998;46:4869-73
Wolf R, Matz H, Orion E, Tuzun B, Tuzun, Y. Dapsone. Dermatol Online J 2002;8:2

Yang W, Tiffany-Castiglioni E. The bipyridyl herbicide paraquat induces proteasome dysfunction in human neuroblastoma SH-SY5Y cells. J Toxicol Environ Health A 2007;70:1849-57

Zelko IN, Mariani TJ, Folz RJ. Superoxide dismutase multigene family: a comparison of the CuZn-SOD (SOD1), Mn-SOD (SOD2), and EC-SOD (SOD3) gene structures, evolution, and expression. Free Radic Biol Med 2002;33: 337-49

Zone JJ. Dermatitis herpetiformis. Curr Probl Dermatol 1991; 3:6-41 